ARTICLE

\title{
Efficient 3D printing via photooxidation of ketocoumarin based photopolymerization
}

\author{
Xiaoyu Zhao (1) ${ }^{1,2}$, Ye Zhao (1) ${ }^{1,2}$, Ming-De Li ${ }^{3}$, Zhong'an Li ${ }^{1}$, Haiyan Peng (1) ${ }^{1,2}$, Tao Xie ${ }^{4}$ \& Xiaolin Xie ${ }^{1,2 凶}$
}

Photopolymerization-based three-dimensional (3D) printing can enable customized manufacturing that is difficult to achieve through other traditional means. Nevertheless, it remains challenging to achieve efficient 3D printing due to the compromise between print speed and resolution. Herein, we report an efficient 3D printing approach based on the photooxidation of ketocoumarin that functions as the photosensitizer during photopolymerization, which can simultaneously deliver high print speed $\left(5.1 \mathrm{~cm} \mathrm{~h}^{-1}\right)$ and high print resolution $(23 \mu \mathrm{m})$ on a common 3D printer. Mechanistically, the initiating radical and deethylated ketocoumarin are both generated upon visible light exposure, with the former giving rise to rapid photopolymerization and high print speed while the latter ensuring high print resolution by confining the light penetration. By comparison, the printed feature is hard to identify when the ketocoumarin encounters photoreduction due to the increased lateral photopolymerization. The proposed approach here provides a viable solution towards efficient additive manufacturing by controlling the photoreaction of photosensitizers during photopolymerization.

\footnotetext{
${ }^{1}$ Key Lab for Material Chemistry of Energy Conversion and Storage, Ministry of Education, School of Chemistry and Chemical Engineering, Huazhong University of Science and Technology (HUST), Wuhan, China. ${ }^{2}$ National Anti-Counterfeit Engineering Research Center, HUST, Wuhan, China. ${ }^{3}$ Key Laboratory for Preparation and Application of Ordered Structural Materials of Guangdong Province, Department of Chemistry, Shantou University (STU), Shantou, China. ${ }^{4}$ State Key Laboratory of Chemical Engineering, College of Chemical and Biological Engineering, Zhejiang University (ZJU), Hangzhou, China.

凶email: hypeng@hust.edu.cn; xlxie@hust.edu.cn
} 
T hree-dimensional (3D) printing represents an additive manufacturing approach in which $3 \mathrm{D}$ objects are created with the aid of computer designs. The most powerful advantage of 3D printing is the capability of producing arbitrarily sophisticated structures that are impossible or very challenging to achieve through other traditional manufacturing methods ${ }^{1-8}$. Furthermore, it can integrate predesigned functions into the 3Dprinted objects as needed ${ }^{9-11}$. So far, 3D printing has enabled a broad range of promising engineering applications, including solar evaporator ${ }^{12}$, energy storage ${ }^{13}$, mechanical dissipation ${ }^{14}$, chemical reactors $^{15}$, microfluidic devices ${ }^{16}$, and biomaterials ${ }^{17,18}$. Nevertheless, the compromise between the print speed and resolution still constrains the print efficiency ${ }^{1,8,9}$.

3D printing is most typically implemented in a layer-wise fashion ${ }^{9}$, despite the successful demonstration of volumetric additive manufacturing reported recently $y^{6,17}$. Among various 3D printing techniques, photopolymerization-based digital light processing (DLP) has attracted particular attention ${ }^{1-4,9,10,12-16 \text {, }}$ due to that the photopolymerization kinetics can be precisely controlled spatially and temporally ${ }^{19-28}$. In this manner, 3D printing can be well controlled with rationally designed monomers ${ }^{7,29-31}$, photoinitiating systems ${ }^{32-34}$, chain transfer agents $^{35}$, oxygen inhibition layers ${ }^{2}$, mobile liquid interfaces ${ }^{1}$, and hardwares $6,17,36$. However, the light transmitted through the solidified polymer layers is highly likely to trigger undesired lateral photopolymerization during the printing of subsequent layers and therefore leads to deteriorated print resolution ${ }^{1,9}$. To confine the light penetration and thus to improve the print resolution, a considerable mass of nonreactive light absorbers (also known as opaquing agents, e.g., Sudan Orange $\mathrm{G}^{3}$, Sudan $\mathrm{I}^{16}$, and Tinuvin $171^{5}$ ) are always required ${ }^{9,37}$. Nonetheless, such improvement comes at the expense of print speed due to that a large amount of light energy is consumed by the nonreactive light absorbers rather than by the photosensitizers ${ }^{8}$. Light absorption by photosensitizers, which is a prerequisite for the conversion of light energy to chemical energy, is critical for photopolymerizationbased 3D printing. Hereto, it remains challenging to develop an efficient 3D printing approach with both high print speed and resolution.

Very recently, Hawker and coworkers have proposed an interesting solution to address the above challenge by using photochromic dyes (e.g., diarylethenes) as reactive light absorbers to selectively confine the light penetration ${ }^{8}$. During $3 \mathrm{D}$ printing, diarylethenes in the closed form absorb light and subsequently transform into the open form. The open form is colorless so that the photosensitizers gain the visible light energy to trigger the photopolymerization. Based on this elegant design, as high as $50 \mathrm{~cm} \mathrm{~h}^{-1}$ of print speed and $100 \mu \mathrm{m}$ of print resolution can be achieved using the one-step printing method. Nonetheless, it remains a great challenge to simultaneously improve the print speed and resolution on common DLP hardware.

Alternatively, it is also rational to resolve the above problem by designing new photoinitiating systems. For instance, by using a reversible addition fragmentation chain transfer (RAFT) agent as the reactive light absorber, Boyer and coworkers ${ }^{38-41}$ have achieved a print speed of $9.1 \mathrm{~cm} \mathrm{~h}^{-1}$ while the print resolution is reported to be $200 \mu \mathrm{m}^{38}$. Allonas and coworkers also reported a resolution of $100 \mu \mathrm{m}$ but with a low print speed of $1.8 \mathrm{~cm} \mathrm{~h}^{-1}$ using other three-component photoinitiating systems ${ }^{42,43}$, wherein the photosensitizer functions as the reactive light absorber. Nonetheless, further increasing the print resolution remains a challenge.

Herein, we demonstrate an efficient 3D printing approach by using ketocoumarin as the photosensitizer (also a reactive light absorber), attributed to (1) ketocoumarins are attractive and distinct photosensitizers with high molar extinction coefficients and high intersystem crossing coefficients ${ }^{44}$, which would promote the light energy efficiency, photoinitiating efficiency, and print speed, (2) ketocoumarins exhibit high photochemical stability in a solidified polymer to prevent photobleaching and thus can afford a low light penetration depth $\mathrm{h}^{45,46}$, which would help increasing the print resolution, and (3) ketocoumarins would generate products with high molar extinction coefficients after photoreaction, which help boosting the print resolution as well. To demonstrate a proof of concept, we used one ketocoumarin compound, i.e., 3,3'-carbonylbis(7-diethylaminocoumarin) (KCD), as the photosensitizer for efficient 3D printing. Excitingly, an attractive print speed $\left(5.1 \mathrm{~cm} \mathrm{~h}^{-1}\right)$ and high print resolution $(23 \mu \mathrm{m})$ were simultaneously achieved on a common bottom-up 3D printer. Furthermore, the light energy efficiency increased 12 times in comparison with traditional systems based on nonreactive light absorbers. Deep insight into the mechanism shows that the photoreaction process of KCD significantly matters for controlling the 3D printing resolution and only the photooxidation process can enable well-defined 3D printing. The initiating radical and deethylated ketocoumarin were simultaneously generated during the photooxidation of KCD. The initiating radical triggered a rapid photopolymerization for facile 3D printing, while the deethylated ketocoumarin ensured high print resolution by confining the light penetration. By comparison, the printed feature could not be identifiable when KCD encountered photoreduction. These findings would open a new door to design efficient additive manufacturing by controlling the photoreaction processes of photosensitizers during photopolymerization.

\section{Results}

Photooxidation products and mechanism. Our efficient 3D printing is realized via the photooxidation of ketocoumarin-based photopolymerization. For proof of concept, we employed commercially available KCD as the photosensitizer that was initially reported by Specht and coworkers in $1979^{47}$. KCD exhibits a high intersystem crossing (ISC) efficiency up to $92 \%{ }^{44}$ and a large maximum molar extinction coefficient $\left(\varepsilon_{\max }\right)$ of $8.8 \times 10^{4} \mathrm{~L} \mathrm{~mol}^{-1}$ $\mathrm{cm}^{-1}$ at $458 \mathrm{~nm}$ (see Supplementary Fig. 1), both of which are beneficial for efficient photoreaction of KCD with a coinitiator upon visible light irradiation ${ }^{44}$. As proposed in Fig. 1a, the photooxidation of KCD by 2-(4-methoxyphenyl)-4,6-bis(trichloromethyl)-1,3,5-triazine (TA) was expected to occur in the presence of water (remained in the solvent and monomer, see Supplementary Table 2), thereby producing the initiating radical (i.e., $\mathrm{TA}^{*}$ ) and deethylated product (i.e., KCD_2). The function of $\mathrm{TA}^{\circ}$ is to initiate a rapid photopolymerization for facile $3 \mathrm{D}$ printing ${ }^{48,49}$, while KCD_2 can confine the light penetration to improve the print resolution due to its large absorption coefficient in the visible light wavelength region $\left(\varepsilon_{\max }=4.3 \times\right.$ $10^{4} \mathrm{~L} \mathrm{~mol}^{-1} \mathrm{~cm}^{-1}$ at $448 \mathrm{~nm}$, see Supplementary Fig. 2).

To start with, the photooxidation products of KCD by TA were identified. Note that although the ketocoumarin/triazine system and other systems based on ketocoumarin or triazine have been proven to be efficient for mediating the visible light photopolymerization ${ }^{50-52}$, the related mechanism and products during the photoreaction have not been fully disclosed, thereby hindering the development of efficient 3D printing. We believe that the photooxidation of $\mathrm{KCD}$ by TA is thermodynamically favorable upon visible light irradiation, based on the calculated free energy $(-0.14 \mathrm{eV})$ of electron transfer using the Rehm-Weller equation ${ }^{49}$ :

$$
\Delta G_{\mathrm{ET}}=e\left[E_{\mathrm{ox} \_\mathrm{KCD}}-E_{\text {red_TA }}\right]-E_{\mathrm{T} \_\mathrm{KCD}}
$$

where $E_{\mathrm{Ox} \_ \text {KCD }}$ and $E_{\text {red_TA }}$ are the oxidation potential of KCD $(+1.06 \mathrm{~V} \text { vs. SCE })^{22}$ and reduction potential of TA $(-1.00 \mathrm{~V}$ 
a

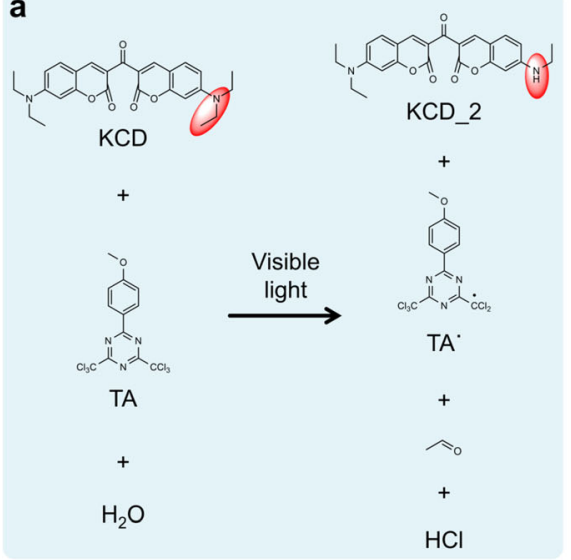

b

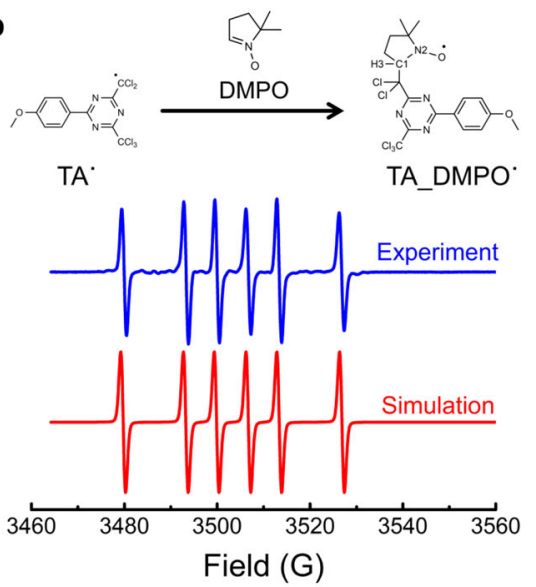

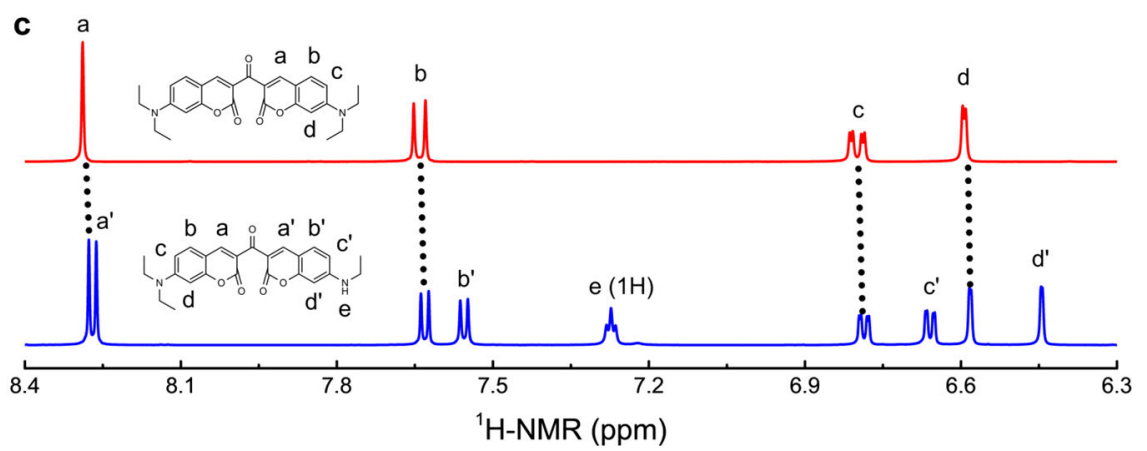

Fig. 1 Photooxidation products of KCD by TA. a Proposed products during the photooxidation of KCD by TA upon visible light irradiation in the presence of water (remained in the monomer and solvent). b Experimental (blue line) and simulated (red line) electron paramagnetic resonance (EPR, EMXmicro, Bruker) signals that were generated during the photooxidation of KCD by TA. c Representative changes of chemical shifts in the ${ }^{1} \mathrm{H}-\mathrm{NMR}$ spectra (Ascend, Bruker) when converting KCD (red line) to KCD_2 (blue line).

vs. SCE $)^{49}$, respectively, and $E_{\mathrm{T} \text { KCD }}$ is the triplet-state energy of $\operatorname{KCD}(2.20 \mathrm{eV})^{44}$. Mechanistically, we suspected that KCD encountered twice oxidation when reacting with TA upon visible light irradiation (see Supplementary Fig. 3), which was supported by the transient absorption spectra and density functional theory calculations (see Supplementary Figs. 4 and 5). Wherein, TA was first reduced by accepting one electron from excited KCD. Subsequently, heterolytic cleavage of the $\mathrm{C}-\mathrm{Cl}$ bond occurred to generate the $\mathrm{TA}^{\circ}$ radical $^{53}$, which was evidenced by electron paramagnetic resonance spectroscopy in the presence of 5,5-dimethyl-1-pyrroline $\mathrm{N}$-oxide (DMPO) as the radical stabilizer (Fig. 1b) ${ }^{22}$. No other radicals were detected. The detected radical shows a $g$-value of 2.0062 along with two hyperfine coupling constants of $20.1 \mathrm{G}$ (H3) and $13.5 \mathrm{G}(\mathrm{N} 2)$, highly consistent with the previous report ${ }^{22}$. Moreover, the produced KCD_2 was isolated and identified by ${ }^{1} \mathrm{H}$ - and ${ }^{13} \mathrm{C}$-nuclear magnetic resonance (NMR) spectroscopy (Fig. 1c, and Supplementary Figs. 6 and 7), as well as highperformance liquid chromatography-high-resolution mass spectrometry (HPLC-HRMS, see Supplementary Figs. 8-18). In addition, the generated acetaldehyde $\left(\mathrm{CH}_{3} \mathrm{CHO}\right)$ was also identified using gas chromatography-mass spectrometry (GC-MS, see Supplementary Figs. 19 and 20).

Print fidelity. As a control, 3D printing based on the photoreduction of KCD was also executed. When KCD is photoreduced by amines, e.g., $N$-phenylglycine (NPG, Fig. 2a) or methyldiethanolamine (MDEA), the generated amino-alkyl radical and ketyl radical result in a large blueshift of the absorption $(\sim 61 \mathrm{~nm})$ compared with $\mathrm{KCD}^{21,22}$, and thus give rise to a significant decrease in the print resolution.
3D printing experiments show that our photopolymerizationbased $3 \mathrm{D}$ printing via photooxidation of KCD could enable high print fidelity upon visible light irradiation. To show a proof of concept, we conducted 3D printing with a common benchtop DLP 3D printer (Fig. 2b). The printing was implemented through the bottom-up process, in which the digital light irradiated the printing resin from the bottom. After printing each layer, the platform moved upward to separate the solidified (cross-linked) polymer parts from the surrounding liquid resin and printing vat. Subsequently, the platform was lowered again into the resin to print the next layer. Since both TA and NPG show negligible absorptions above the wavelength of $420 \mathrm{~nm}$ (see Supplementary Fig. 21), we added a band-pass filter above the digital light projector to confine the light wavelength $(420-780 \mathrm{~nm})$ during printing. Under such a circumstance, only KCD can be directly photoexcited. A monomer mixture composed of $\mathrm{N}, \mathrm{N}$-dimethylacrylamide (DMAA), trimethylolpropane ethoxylate triacrylate (TMPEOTA), and pentaerythritol tetraacrylate (PETTA) was used during 3D printing. DMAA can help to dissolve KCD, TA, and NPG. TMPEOTA and PETTA were used to provide crosslinked polymer parts with sufficient strength prior to postcure ${ }^{9}$. However, adhesion of the printed polymer parts to the printing vat should be avoided ${ }^{1,2}$. In the typical $3 \mathrm{D}$ printing method, the weight ratio of DMAA, TMPEOTA, and PETTA was optimized to be 3.5:5.0:1.5, which however can be changed depending on the KCD concentration (see Supplementary Fig. 22). Moreover, note that monomers such as 1,6-hexanediol diacrylate (HDDA), poly (ethylene glycol) diacrylate (PEGDA, $M_{\mathrm{n}} \sim 200$ ), and bisphenol A glycerolate diacrylate (Epoxy acrylate) can also be used for successful 3D printing (see Supplementary Fig. 23). Postcure was conducted to consume the unreacted monomers in the polymer 

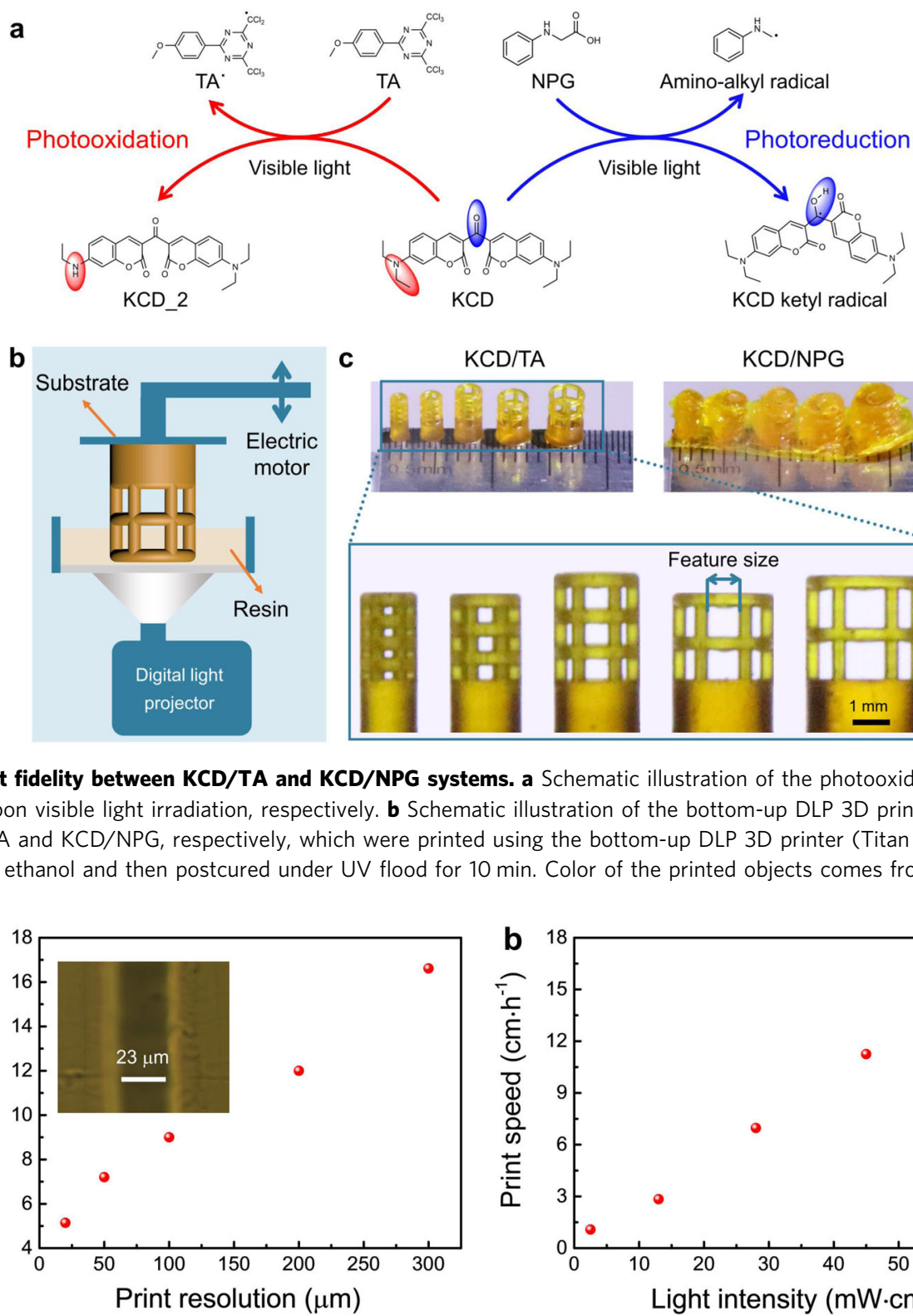

KCD/TA

KCD/NPG

Fig. 2 Comparison of print fidelity between KCD/TA and KCD/NPG systems. a Schematic illustration of the photooxidation of KCD by TA and photoreduction by NPG upon visible light irradiation, respectively. b Schematic illustration of the bottom-up DLP 3D printing. $\mathbf{c}$ 3D-printed objects photomediated by KCD/TA and KCD/NPG, respectively, which were printed using the bottom-up DLP 3D printer (Titan 2, Kudo3D). The 3D-printed objects were cleaned with ethanol and then postcured under UV flood for $10 \mathrm{~min}$. Color of the printed objects comes from KCD and KCD_2.
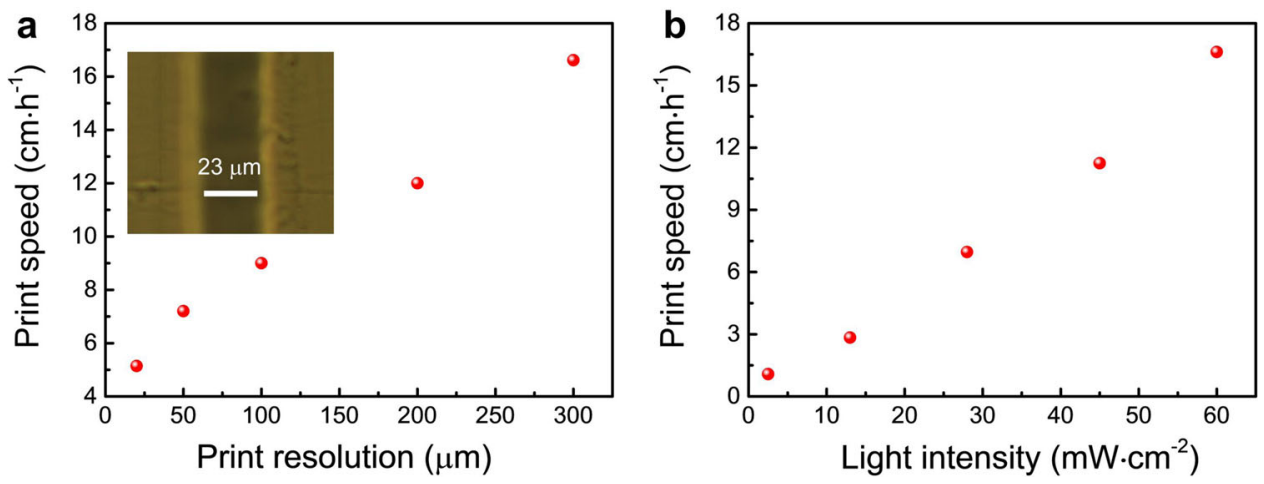

Fig. 3 High print resolution and speed enabled by photooxidation of KCD. Print speed against a print resolution and $\mathbf{b}$ light intensity of $420-780 \mathrm{~nm}$ light. Inset of a Optical microscope image (Axio Scope A1, Carl Zeiss) of the 3D-printed line photomediated by the KCD/TA system.

parts as suggested ${ }^{9,40}$. The double-bond conversion of the printing resin was increased from $36 \%$ to $95 \%$ upon postcure (see Supplementary Fig. 24). The tensile strength and tensile modulus of printed objects were increased from $21 \pm 2 \mathrm{MPa}$ and $112 \pm 10 \mathrm{MPa}$ to $40 \pm 3 \mathrm{MPa}$ and $260 \pm 15 \mathrm{MPa}$, respectively, while the elongation at break was decreased from $36 \pm 3 \%$ to $29 \pm 2 \%$ (see Supplementary Fig. 25, Supplementary Table 3).

We initially evaluated the print fidelity by printing an array of open cylinders with square lattices. The diameter of each grid line of the square lattice was set as $400 \mu \mathrm{m}$ in the computer-aided design model, while the feature size of the square lattice was varied from 200 to $1000 \mu \mathrm{m}$ in $200 \mu \mathrm{m}$ increments. Excitingly, the printed open cylinders agree well with those predesigned when employing our 3D printing based on the photooxidation of KCD (Fig. 2c and Supplementary Fig. 26). By comparison, the print fidelity showed a dramatic decrease when KCD encountered photoreduction in the presence of amines (e.g., NPG and MDEA). In addition, no printed feature structure can be identified if $3 \mathrm{D}$ printing was photomediated by commercially available photoinitiating systems such as Irgacure 784/benzoyl peroxide and camphorquinone/NPG (see Supplementary Fig. 27), attributed to the increased lateral photopolymerization (see Supplementary Note 1). It is well known that the undesired lateral photopolymerization results in microparticulate solids in the printing resin, which then tend to connect all the printed parts and thus reduce the print fidelity ${ }^{1}$.

Print resolution and speed. Both high print resolution and print speed have been successfully enabled in our photopolymerization-based $3 \mathrm{D}$ printing that is mediated by the photooxidation of KCD. In the absence of KCD or light irradiation, 3D printing was very hard to proceed due to the negligible monomer conversion under those conditions (see Supplementary Fig. 28). We then evaluated the print resolution by printing a line with a width of 1 pixel $(23 \mu \mathrm{m})^{54}$, showing a high print resolution $(23 \mu \mathrm{m})$ achieved upon exposure to $60 \mathrm{~mW} \mathrm{~cm}^{-2}$ of $420-780 \mathrm{~nm}$ light irradiation (Fig. 3a). 

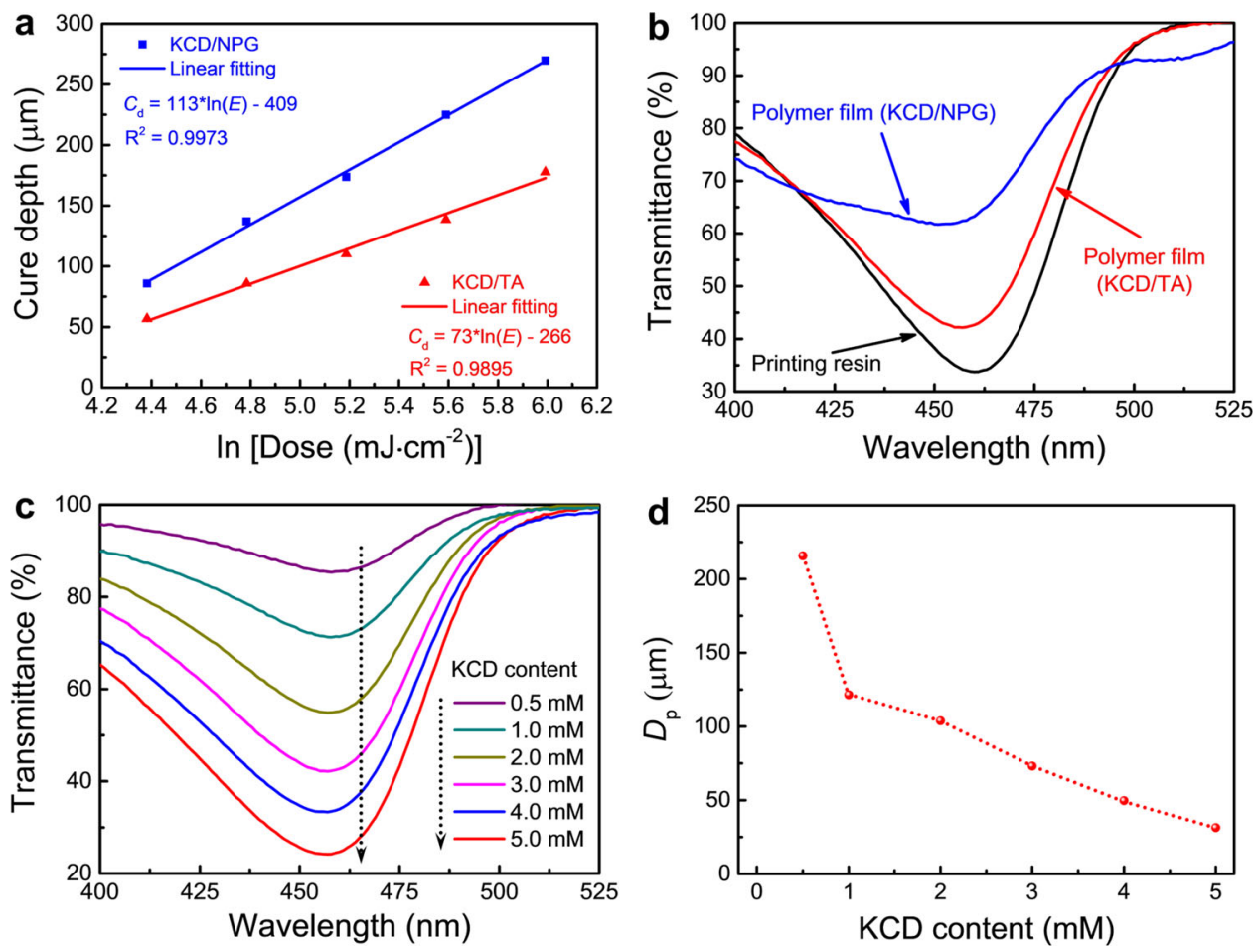

Fig. 4 Performance of the printing resin. a Cure depth $\left(C_{d}\right)$ of the printing resin against irradiation dose $(E)$ at $460 \mathrm{~nm}$. $\mathbf{b}$ Light transmittance measured by an ultraviolet-visible spectrometer (Revolution 220, Thermo Fisher Scientific) of the polymer films and printing resin with a thickness of $20 \mu \mathrm{m}$. $\mathbf{c}$ Light transmittance of the polymer films with a thickness of $20 \mu \mathrm{m}$ against KCD content. $\mathbf{d} D_{\mathrm{p}}$ against KCD content.

Meanwhile, an attractive print speed of $5.1 \mathrm{~cm} \mathrm{~h}^{-1}$ can be achieved by printing each $20 \mu \mathrm{m}$ thick layer in $1.4 \mathrm{~s}$. It is worth noting that the print speed does not count the moving time of the platform ${ }^{40,41}$. In addition, the print speed was increased from 5.1 to $16.6 \mathrm{~cm} \mathrm{~h}^{-1}$ when the print resolution was varied from 23 to $300 \mu \mathrm{m}$ (Fig. 3a), and it was decreased from 16.6 to $1.1 \mathrm{~cm} \mathrm{~h}^{-1}$ when the light intensity was decreased from 60 to $2.5 \mathrm{~mW} \mathrm{~cm}^{-2}$ (Fig. 3b).

Elegant designs of hardware and printing resins have been concerned to promote the print speed and print resolution. With respect to the hardware, for instance, DeSimone et al. ${ }^{2}$ and Mirkin et al. ${ }^{1}$ developed continuous 3D printing setups to increase the print speed to $2.5 \mathrm{~cm} \mathrm{~h}^{-1}$ (resolution: $50 \mu \mathrm{m}$ ) and $43.2 \mathrm{~cm} \mathrm{~h}^{-1}$ (resolution: $300 \mu \mathrm{m}$ ), respectively. Zheng and coworkers used the liquid-crystal on-silicon chip (LCoS) and lens to reduce the pixel size of the digital light engine and thus increased the print resolution to $10 \mu \mathrm{m}^{55,56}$. Lee and coworkers used the lens to increase the print speed and resolution to $32.7 \mathrm{~cm} \mathrm{~h}^{-1}$ and $100 \mu \mathrm{m}$, respectively ${ }^{57}$. Distinctly, in the aspect of printing resins, the highest print resolution $(18 \mu \mathrm{m})$ was reported by Nordin and coworkers with a print speed of $2.4 \mathrm{~cm} \mathrm{~h}^{-1}$ using a common DLP 3D printer ${ }^{58}$. Furthermore, the $3 \mathrm{D}$ printing systems based on RAFT agents ${ }^{38-41}$ and threecomponent photoinitiating systems $\mathrm{s}^{42,43}$ produced print speeds/ resolutions of $9.1 \mathrm{~cm} \mathrm{~h}^{-1} / 200 \mu \mathrm{m}$ and $1.8 \mathrm{~cm} \mathrm{~h}^{-1 / 100 \mu \mathrm{m} \text { on a }}$ common DLP 3D printer. In contrast, our KCD/TA system can produce a 12-times higher light energy efficiency $(\varphi=24 \%)$ than that of the commonly used nonreactive light-absorberbased system $(\varphi=2 \%$, see Supplementary Notes 2 and 3$)$, leading to an attractive print speed of $5.1 \mathrm{~cm} \mathrm{~h}^{-1}$ and a high print resolution of $23 \mu \mathrm{m}$. Note that the print speed is also 2.1 times faster than that of the above mentioned state-of-theart $3 \mathrm{D}$ printing approaches using the common DLP hardware while maintaining comparable print resolution (see Supplementary Table 4).
Performance of the printing resin. High print resolution results from the decreased light penetration depth $\left(D_{\mathrm{p}}\right)$ during 3D printing, which can be calculated from the following equation?

$$
C_{\mathrm{d}}=D_{\mathrm{p}} \times \ln (E)-D_{\mathrm{p}} \times \ln \left(E_{\mathrm{c}}\right)
$$

where $C_{\mathrm{d}}$ and $E$ denote the cure depth and irradiation dose during $3 \mathrm{D}$ printing, respectively, and $E_{\mathrm{c}}$ is the critical irradiation dose of the printing resin. As illustrated in Fig. 4a, despite the identical KCD concentration $(3 \mathrm{mM})$, the calculated $D_{\mathrm{p}}(73 \mu \mathrm{m})$ of the $\mathrm{KCD} / \mathrm{TA}$ system is 1.5 times smaller than that of the KCD/NPG system, promising much better print resolution. We believe that this diffference is dominated by the light transmittance of the solidified polymer layers. To provide a quantitative proof, we characterized the light transmittance of polymer films in a thickness of $20 \mu \mathrm{m}$. The thickness was identical to that of each printed layer during $3 \mathrm{D}$ printing. The results show that the light transmittance of all solidified polymer films is increased compared to the liquid printing resin because of the photoreaction of $\mathrm{KCD}$ (Fig. $4 \mathrm{~b}$ ). Within the visible light wavelength region, the minimum light transmittance is $62 \%$ and $42 \%$ for the polymer films (thickness: $20 \mu \mathrm{m}$ ) photomediated by KCD/NPG and KCD/ TA, respectively, indicating a decreased light penetration for the latter. In addition, because of the decreased light transmittance of the polymer film with an increase in KCD concentration (Fig. 4c), the $D_{\mathrm{p}}$ was found to be decreased significantly (Fig. $4 \mathrm{~d}$, Supplementary Fig. 29).

Although visible light-sensitive printing resins are of high reactivity, our printing resin can be stored in a brown-color vial under dark or ambient conditions for at least 7 days without detectable changes in the appearance, ground-state absorption, or viscosity (see Supplementary Figs. 30 and 31). However, when the printing resin was stored in a transparent vial under ambient conditions, photopolymerization will occur and induce a dramatic increase in the viscosity. 


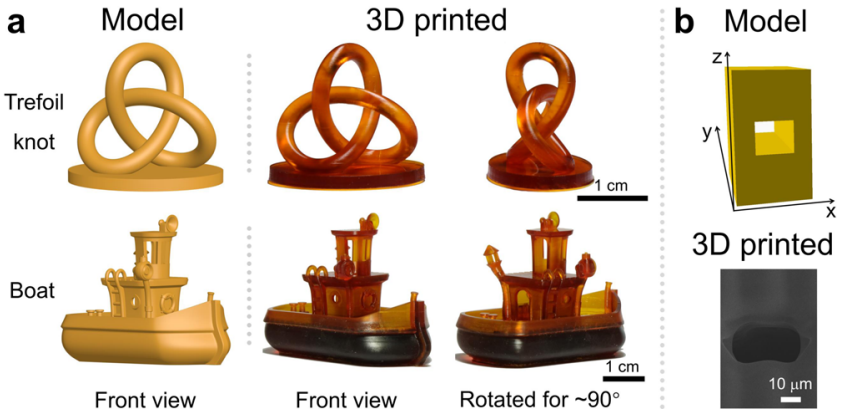

Fig. 5 Models and corresponding 3D-printed objects. a Pictures to show the 3D-printed trefoil knot and boat based on the KCD/TA system. The 3Dprinted objects were rinsed with ethanol and postcured under UV flood ( $50 \mathrm{~mW} \mathrm{~cm}^{-2}$ ) for $10 \mathrm{~min}$. Color of the printed objects comes from KCD and KCD_2. $\mathbf{b}$ Model of the predesigned microscale hole and the corresponding SEM image based on the KCD/TA system.

Printed 3D objects. We can further print 3D-sophisticated objects based on our efficient 3D printing through the $\mathrm{KCD} / \mathrm{TA}$ system-mediated photopolymerization. Fig. 5a shows the successful printing of a trefoil knot (designed according to the reported method ${ }^{59}$ ) and a boat (model authorized by vandragon_de from the MakerBot Thingiverse community). The print fidelity is very high in both cases, and the throughputs of trefoil knot and boat were 1.8 and $10.5 \mathrm{~cm}^{3} \mathrm{~h}^{-1}$, respectively. To further confirm the high print fidelity for our method, we designed a tiny hole with $20 \mu \mathrm{m}$ in height and $23 \mu \mathrm{m}$ (1 pixel) in width, showing that high-fidelity printing can be achieved with the precisely controlled height, despite increasing the width to $35 \mu \mathrm{m}$ (1.5 pixels) due to the scattering of light (Fig. $5 \mathrm{~b})^{58}$.

We further demonstrated that KCD can also be photooxidized by onium salts (e.g., iodonium ${ }^{60}$ and sulfonium ${ }^{51}$ ), and thus the proposed paradigm here opens the door to design efficient $3 \mathrm{D}$ printing systems by controlling the photoreaction of photosensitizers during photopolymerization.

\section{Discussion}

In summary, we proposed and demonstrated an efficient 3D printing approach via the photooxidation of ketocoumarin (e.g., KCD) based photopolymerization, which enabled both high print speed $\left(5.1 \mathrm{~cm} \mathrm{~h}^{-1}\right)$ and high print resolution $(23 \mu \mathrm{m})$. The print speed was much faster than the state-of-the-art 3D printing approaches using the common bottom-up DLP 3D printer without compromising the print resolution. The light energy efficiency was also 12 times higher than traditional systems containing nonreactive light absorbers. Mechanistically, the produced free radicals led to fast photopolymerization for facile 3D printing, while the deethylated KCD confined the light penetration for improving the print resolution. By comparison, the print resolution was dramatically decreased when KCD encountered photoreduction due to that the increased light transmittance of the printed polymer parts led to increased lateral photopolymerization. The proposed 3D printing approach here based on the photooxidation of ketocoumarin paves the way for efficient additive manufacturing by controlling the photoreaction of photosensitizers during photopolymerization.

\footnotetext{
Methods

Materials. N,N-Dimethylacrylamide (DMAA, purity: 98\%), 5,5-dimethyl-1-pyrroline $N$-oxide (DMPO, purity: $97 \%$ ), 2-(4-methoxyphenyl)-4,6-bis(trichloromethyl)-1,3,5-triazine (TA, purity: $98 \%$ ), pentaerythritol tetraacrylate (PETTA, purity: $>80 \%$ ), and 1,6-hexanediol diacrylate (HDDA, purity: $>85 \%$ ) were purchased from TCI Chemicals. Toluene (spectrometric grade, purity: 99.7\%), $\mathrm{N}$ phenylglycine (NPG, purity: $97 \%$ ), acetonitrile (purity: AR), trimethylolpropane ethoxylate triacrylate (TMPEOTA, average $M_{\mathrm{n}} \sim 692$ ), dichloromethane (DCM,
}

purity: AR), ethylacetate (EA, purity: AR), ammonia water (liquid chromatography/mass spectrometry (LC/MS) grade), and poly(ethylene glycol) diacrylate (PEGDA, $M_{\mathrm{n}} \sim 200$ ) were received from Aladdin, China. Bisphenol A glycerolate diacrylate (Epoxy acrylate) was received from Sigma-Aldrich. Camphorquinone (CQ, purity: 99\%) was received from Alfa Aesar. Irgacure 784 (purity: 99\%) was obtained from Alfa Chem, China. Benzoyl peroxide (BPO) and methyldiethanolamine (MDEA, purity: 99\%) were received from Innochem Co., Ltd., China. 3,3'Carbonylbis(7-diethylaminocoumarin) (KCD, purity: 99\%) was obtained from Acros Organics. DMSO- $d_{6}$ (purity: 99.8 atom\%D) was purchased from J\&K Scientific, China. Acetonitrile ( $\mathrm{AcCN}$ ) and water in LC/MS grade for highperformance liquid chromatography-high-resolution mass spectrometry (HPLC-HRMS) characterization were purchased from Thermo Fisher Scientific. NPG was recrystallized from hot water in the presence of activated charcoal before use, while other chemicals were used directly without further purification.

Gas chromatography-mass spectrometry (GC-MS). Acetaldehyde $\left(\mathrm{CH}_{3} \mathrm{CHO}\right)$ was expected to generate during the photooxidation of KCD by TA, which was characterized using GC-MS. First, KCD $(1 \mathrm{mM})$ and TA $(1 \mathrm{mM})$ were homogeneously dissolved in toluene upon bulk-ultrasonication at $298 \mathrm{~K}$ for $30 \mathrm{~min}$. Then the homogenous solution was purged with argon gas for $30 \mathrm{~min}$ to remove the oxygen, followed by white light irradiation (wavelength: $420-780 \mathrm{~nm}$ ) for $30 \mathrm{~min}$ with an intensity of $20.0 \mathrm{~mW} \mathrm{~cm}-2$. After removing the solid by filtration, the residue was characterized using a GC/MS setup (5977B MSD, Agilent Technology, United States) with the Ultra Inert column (HP-5MS). The applied parameters were set as follows: carrier gas, high-purity helium; injection port temperature, $573 \mathrm{~K}$; distribution ratio of flow, 10/1; injection sample volume, $1 \mu \mathrm{L}$; carrier gas

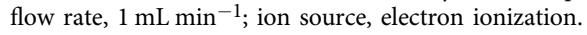

High-performance liquid chromatography-high-resolution mass spectrometry (HPLC-HRMS). The main products generated during the photooxidation of KCD $(1 \mathrm{mM})$ by TA $(1 \mathrm{mM})$ in toluene were identified using HPLC-HRMS on an LC/ MS setup (Orbitrap, Thermo Fisher Scientific, United States) with a C18 column. Prior to characterization, toluene was removed under vacuum and the residual was dissolved in AcCN. The mobile phase was the mixture of $\mathrm{AcCN}$ and water in $\mathrm{LC} /$ MS grade. The $\mathrm{pH}$ of the mobile phase was tuned to be 9.0 with ammonia water in order to improve the chromatography efficiency. The flow rate was $0.2 \mathrm{~mL} \mathrm{~min}^{-1}$. The volume fraction of AcCN in the mobile phase was optimized to be $30 \%$ from 0 to $12 \mathrm{~min}$, which was then increased to $95 \%$ from 12 to $20 \mathrm{~min}$. The ion source was atmospheric pressure chemical ionization. Chemical species in the mobile phase were probed by light with wavelengths of 460 and $226 \mathrm{~nm}$, respectively, at the temperature of $303 \mathrm{~K}$.

Nuclear magnetic resonance (NMR) spectroscopy. KCD_2 as one of the primary photooxidation products was also identified by NMR after isolation through silica column chromatography (eluent: DCM/EA with a volume ratio of 50/1). Both ${ }^{1} \mathrm{H}$ - and ${ }^{13} \mathrm{C}-\mathrm{NMR}$ characterizations were conducted on a $600 \mathrm{MHz}$ NMR spectrometer (Ascend, Bruker, Germany) at $298 \mathrm{~K}$. DMSO- $d_{6}$ was used as the solvent.

Ground-state absorption spectroscopy. Ground-state absorptions were characterized on one ultraviolet-visible (UV-vis) spectrometer (Revolution 220, Thermo Fisher Scientific, United States). AcCN was used as the solvent. The concentrations of KCD, KCD_2, TA and NPG were controlled to be $10 \mu \mathrm{M}$.

Characterization of water content. The water contents in toluene, DMAA, TMPEOTA, and PETTA were measured using a Karl Fischer moisture titrator (870 KF Titrino plus, Metrohm, Switzerland).

Electron paramagnetic resonance (EPR) spectroscopy. EPR spectroscopy was conducted on a spectrometer (EMXmicro, Bruker, Germany) to identify the radical species during photoreaction. A homogeneous solution was prepared by dissolving KCD $(175 \mu \mathrm{M})$, TA $(3.5 \mathrm{mM})$, and DMPO (the radical capturing agent, $3.5 \mathrm{mM})$ in toluene upon bulk ultrasonication $(298 \mathrm{~K}, 30 \mathrm{~min})$. The concentrations of $\mathrm{KCD}$ and TA were maintained to be identical to that during the nanosecond transient absorption characterization, respectively. Prior to measurement, oxygen was removed by continuous argon gas purge. Then, the solution was loaded in an EPR columnar quartz cell that was subsequently placed into the spectrometer. Radicals were generated upon exposure to light irradiation under a high-pressure mercury lamp and their signals were in situ recorded. The light wavelength was confined to be $420-780 \mathrm{~nm}$. The applied parameters were set as follows: center field, 3514.2 Gauss (G); sweep width, $100 \mathrm{G}$; microwave power, $2.0 \mathrm{~mW}$; modulation frequency, $100 \mathrm{kHz}$; modulation amplitude, $1 \mathrm{G}$; receiver gain, $30 \mathrm{~dB}$; conversion time, $15 \mathrm{~ms}$; time constant, $0.01 \mathrm{~ms}$. The computer program EasySpin ${ }^{61}$ was employed to fit the recorded signals.

Photopolymerization kinetics. Photopolymerization kinetics upon visible light irradiation (wavelength: $460 \mathrm{~nm}$ ) was analyzed by real-time Fourier transforminfrared spectroscopy (RT-FTIR, Vertex 80, Bruker, Germany). Plastic spacers were placed between two $\mathrm{NaCl}$ salt plates to control the sample thickness $(20 \mu \mathrm{m}$, which 


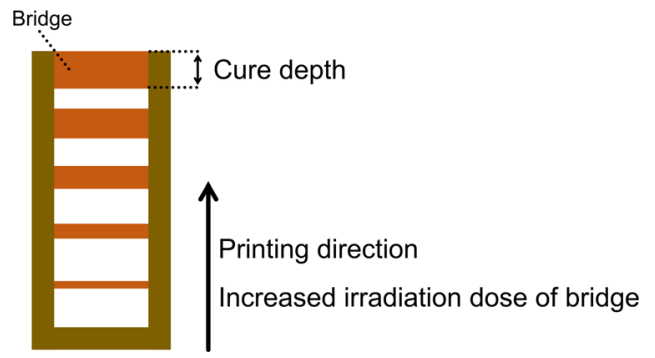

Fig. 6 Schematic illustration of the cure-depth characterization. A bridge model was printed for characterization by increasing the irradiation dose along the printing direction.

was the same as the layer thickness during $3 \mathrm{D}$ printing). The light intensity was $1.2 \mathrm{~mW} \mathrm{~cm}^{-2}$ (identical to the light intensity when characterizing the penetration depth and critical irradiation dose) after considering the light transmittance of the $\mathrm{NaCl}$ plate. The absorption band associated with the $\mathrm{C}-\mathrm{H}$ rocking vibration of vinyl groups ${ }^{22}$ was monitored to characterize the double-bond conversion $(\alpha)$ of acrylates ${ }^{62}$ :

$$
\alpha=1-\frac{A_{\mathrm{t}}}{A_{0}}
$$

where $A_{\mathrm{t}}$ and $A_{0}$ represented the absorption areas at the irradiation time $t$ and before light irradiation, respectively.

Light transmittance of polymer films. Light transmittance of the photomediated polymer films was measured on a UV-vis spectrometer (Revolution 220, Thermo Fisher Scientific, United States). Before characterization, the printing resin was injected into a glass cell with a gap of $20 \mu \mathrm{m}$ (same as the layer thickness during $3 \mathrm{D}$ printing), followed by light irradiation from the 3D printer (Titan 2, Kudo3D, United States) with a band-pass filter to confine the light wavelength (420-780 nm). The light intensity was $60 \mathrm{~mW} \mathrm{~cm}^{-2}$, and the irradiation duration was $2 \mathrm{~s}$, which were identical to the intensity and duaration used to print each layer during 3D printing. To obtain the exact light transmittance of the polymer film, the light absorbance of each glass slide was measured and subtracted as the background. The concentrations of KCD were $0.5,1.0,2.0,3.0,4.0$, and $5.0 \mathrm{mM}$, respectively, while the concentrations of NPG and TA were fixed at $50.0 \mathrm{mM}$, respectively.

Characterization of the penetration depth. To characterize the penetration depth $\left(D_{\mathrm{p}}\right)$, the cure depth of the printing resin was measured against the irradiation dose. The bridge model was used (Fig. 6) ${ }^{63}$, and the cure depth was measured using an optical microscope (Axio Scope A1, Carl Zeiss).

3D printing. 3D printing was implemented on a cost-effective benchtop DLP printer (Titan 2, Kudo3D, United States). A band-pass filter was employed to confine the incident light so that the output light was $420-780 \mathrm{~nm}$, and the intensity was $60 \mathrm{~mW} \mathrm{~cm}^{-2}$. The 3D-printed objects were washed with ethanol and postcured under UV light $\left(50 \mathrm{~mW} \mathrm{~cm}^{-2}\right)$ for $10 \mathrm{~min}$.

Field-effect scanning electron microscopy. To determine the resolution of the 3D-printed microscale objects, samples were characterized by a field-effect scanning electron microscope (SU8010, Hitachi, Japan). Before characterization, samples were adhered to metal plates using conductive tapes, and then a thin layer of gold was sputtered on the surface to improve the image quality during measurement.

\section{Data availability}

All the relevant data supporting the findings of this study are available from the corresponding author upon request.

Received: 17 August 2020; Accepted: 14 April 2021; Published online: 17 May 2021

\section{References}

1. Walker, D. A., Hedrick, J. L. \& Mirkin, C. A. Rapid, large-volume, thermally controlled 3D printing using a mobile liquid interface. Science 366, 360-364 (2019).
2. Tumbleston, J. R. et al. Continuous liquid interface production of 3D objects Science 347, 1349-1352 (2015).

3. Kotz, F. et al. Three-dimensional printing of transparent fused silica glass. Nature 544, 337-339 (2017).

4. Eckel, Z. C. et al. Additive manufacturing of polymer-derived ceramics. Science 351, 58-62 (2016).

5. Chen, X. et al. High-speed 3D printing of millimeter-size customized aspheric imaging lenses with sub $7 \mathrm{~nm}$ surface roughness. Adv. Mater. 30, 1705683 (2018).

6. Kelly, B. E. et al. Volumetric additive manufacturing via tomographic reconstruction. Science 363, 1075-1079 (2019).

7. Deng, S., Wu, J., Dickey, M. D., Zhao, Q. \& Xie, T. Rapid open-air digital light 3D printing of thermoplastic polymer. Adv. Mater. 31, 1903970 (2019).

8. Dolinski, N. D. et al. Solution mask liquid lithography (SMaLL) for one-step, multimaterial 3D printing. Adv. Mater. 30, 1800364 (2018).

9. Ligon, S. C., Liska, R., Stampfl, J., Gurr, M. \& Mülhaupt, R. Polymers for 3D printing and customized additive manufacturing. Chem. Rev. 117, 10212-10290 (2017).

10. Elder, B. et al. Nanomaterial patterning in 3D printing. Adv. Mater. 32, 1907142 (2020).

11. Shafranek, R. T. et al. Stimuli-responsive materials in additive manufacturing. Prog. Polym. Sci. 93, 36-67 (2019).

12. Wu, L. et al. Highly efficient three-dimensional solar evaporator for high salinity desalination by localized crystallization. Nat. Commun. 11, 521 (2020).

13. Egorov, V., Gulzar, U., Zhang, Y., Breen, S. \& O'Dwyer, C. Evolution of 3D printing methods and materials for electrochemical energy storage. $A d v$. Mater. 32, 2000556 (2020).

14. Traugutt, N. A. et al. Liquid-crystal-elastomer-based dissipative structures by digital light processing 3D printing. Adv. Mater. 32, 2000797 (2020).

15. Parra-Cabrera, C., Achille, C., Kuhn, S. \& Ameloot, R. 3D printing in chemical engineering and catalytic technology: Structured catalysts, mixers and reactors. Chem. Soc. Rev. 47, 209-230 (2018).

16. Bhattacharjee, N., Urrios, A., Kang, S. \& Folch, A. The upcoming 3D-printing revolution in microfluidics. Lab Chip 16, 1720-1742 (2016).

17. Bernal, P. N. et al. Volumetric bioprinting of complex living-tissue constructs within seconds. Adv. Mater. 31, 1904209 (2019).

18. Ge, Q. et al. 3D printing of highly stretchable hydrogel with diverse UV curable polymers. Sci. Adv. 7, eaba4261 (2021).

19. Zhao, Y. et al. Crosstalk-free patterning of cooperative-thermoresponse images by the synergy of the AIEgen with the liquid crystal. Angew. Chem. Int. Ed. 59, 10066-10072 (2020).

20. $\mathrm{Hu}, \mathrm{Y}$. X. et al. Construction of supramolecular liquid-crystalline metallacycles for holographic storage of colored images. J. Am. Chem. Soc. 142, 6285-6294 (2020).

21. Peng, H. Y. et al. Monochromatic visible light "photoinitibitor": Janus-faced initiation and inhibition for storage of colored 3D images. J. Am. Chem. Soc. 136, 8855-8858 (2014)

22. Zhao, X. Y. et al. Effect of ketyl radical on the structure and performance of holographic polymer/liquid-crystal composites. Sci. China Mater. 62, 1921-1933 (2019)

23. Zhu, J., Zhang, Q., Yang, T., Liu, Y. \& Liu, R. 3D printing of multi-scalable structures via high penetration near-infrared photopolymerization. Nat. Commun. 11, 3462 (2020).

24. McCarthy, B. G. et al. Structure-property relationships for tailoring phenoxazines as reducing photoredox catalysts. J. Am. Chem. Soc. 140, 5088-5101 (2018).

25. Aguirre-Soto, A., Lim, C. H., Hwang, A. T., Musgrave, C. B. \& Stansbury, J. W. Visible-light organic photocatalysis for latent radical-initiated polymerization via $2 \mathrm{e}(-) / 1 \mathrm{H}(+)$ transfers: Initiation with parallels to photosynthesis. J. Am. Chem. Soc. 136, 7418-7427 (2014).

26. Yagci, Y., Jockusch, S. \& Turro, N. J. Photoinitiated polymerization: Advances, challenges, and opportunities. Macromolecules 43, 6245-6260 (2010).

27. Hoyle, C. E. \& Bowman, C. N. Thiol-ene click chemistry. Angew. Chem. Int Ed. 49, 1540-1573 (2010).

28. Dong, Z. et al. 3D printing of inherently nanoporous polymers via polymerization-induced phase separation. Nat. Commun. 12, 247 (2021).

29. Gräfe, D. et al. Adding chemically selective subtraction to multi-material 3D additive manufacturing. Nat. Commun. 9, 2788 (2018).

30. Wallin, T. J. et al. 3D printable tough silicone double networks. Nat. Commun 11, 4000 (2020).

31. Zhu, G., Hou, Y., Xu, J. \& Zhao, N. Reprintable polymers for digital light processing 3D printing. Adv. Funct. Mater. 31, 2007173 (2021).

32. Al Mousawi, A. et al. Carbazole scaffold based photoinitiator/photoredox catalysts: Toward new high performance photoinitiating systems and application in LED projector 3D printing resins. Macromolecules 50, 2747-2758 (2017).

33. Schwartz, J. J. \& Boydston, A. J. Multimaterial actinic spatial control 3D and 4D printing. Nat. Commun. 10, 791 (2019). 
34. de Beer, M. P. et al. Rapid, continuous additive manufacturing by volumetric polymerization inhibition patterning. Sci. Adv. 5, eaau8723 (2019).

35. Seidler, K. et al. Vinyl sulfonate esters: Efficient chain transfer agents for the 3D printing of tough photopolymers without retardation. Angew. Chem. Int. Ed. 57, 9165-9169 (2018).

36. Zhang, Y. et al. Continuous 3D printing from one single droplet. Nat. Commun. 11, 4685 (2020)

37. Ahn, D., Stevens, L. M., Zhou, K. \& Page, Z. A. Rapid high-resolution visible light 3D printing. ACS Cent. Sci. 6, 1555-1563 (2020).

38. Lee, K., Corrigan, N. \& Boyer, C. Rapid high-resolution 3D printing and surface functionalization via type I photoinitiated RAFT polymerization. Angew. Chem. Int. Ed. 133, 8839-8850 (2021).

39. Bagheri, A. et al. Oxygen tolerant PET-RAFT facilitated 3D printing of polymeric materials under visible LEDs. ACS Appl. Polym. Mater. 2, 782-790 (2020).

40. Zhang, Z., Corrigan, N., Bagheri, A., Jin, J. \& Boyer, C. A versatile 3D and 4D printing system through photocontrolled RAFT polymerization. Angew. Chem. Int. Ed. 58, 17954-17963 (2019).

41. Bagheri, A. et al. 3D printing of polymeric materials based on photo-RAFT polymerization. Polym. Chem. 11, 641-647 (2020).

42. Metral, B., Bischoff, A., Ley, C., Ibrahim, A. \& Allonas, X. Photochemical study of a three-component photocyclic initiating system for free radical photopolymerization: Implementing a model for digital light processing 3D printing. ChemPhotoChem 3, 1109-1118 (2019).

43. Nejadebrahim, A. et al. A new safranin based three-component photoinitiating system for high resolution and low shrinkage printed parts via digital light processing. RSC Adv. 9, 39709-39720 (2019).

44. Specht, D. P., Martic, P. A. \& Farid, S. Ketocoumarins: A new class of triplet sensitizers. Tetrahedron 38, 1203-1211 (1982).

45. Wang, Z. S. et al. A high-light-harvesting-efficiency coumarin dye for stable dye-sensitized solar cells. Adv. Mater. 19, 1138-1141 (2007).

46. Cao, D. et al. Coumarin-based small-molecule fluorescent chemosensors. Chem. Rev. 119, 10403-10519 (2019).

47. Specht, D. P. \& Farid, S. Y. Light-sensitive compositions with 3-substituted coumarin compounds as spectral sensitizers. US Patent US4147552 (1979).

48. Christmann, J., Allonas, X., Ley, C., Ibrahim, A. \& Croutxé-Barghorn, C. Triazine-based type-II photoinitiating system for free radical photopolymerization: Mechanism, efficiency, and modeling. Macromol. Chem. Phys. 218, 1600597 (2017).

49. Grotzinger, C., Burget, D., Jacques, P. \& Fouassier, J. P. Photopolymerization reactions initiated by a visible light photoinitiating system: Dye/amine/bis (trichloromethyl)-substituted-1,3,5-triazine. Macromol. Chem. Phys. 202, 3513-3522 (2001).

50. Zhang, J. et al. A known photoinitiator for a novel technology: 2-(4methoxystyryl)-4,6-bis(trichloromethyl)-1,3,5-triazine for near UV or visible LED. Polym. Chem. 5, 6019-6026 (2014).

51. Toba, Y., Usui, Y., Konishi, T., Ito, O. \& Uesugi, T. Visible light polymerization of acrylate using dialkylphenacylsulfonium butyltriphenylborate initiators: Effect of the reduction potential of the sulfonium cation on the polymerization. Macromolecules 32, 6545-6551 (1999).

52. Salmi, H., Tar, H., Ibrahim, A., Ley, C. \& Allonas, X. Ketocoumarin/triazine/ thiol as new high speed photoinitiating system for free radical polymerization under visible light in aerated media. Eur. Polym. J. 49, 2275-2279 (2013).

53. Pohlers, G., Scaiano, J. C., Step, E. \& Sinta, R. Ionic vs free radical pathways in the direct and sensitized photochemistry of 2-(4'-methoxynaphthyl)-4,6-bis (trichloromethyl)-1,3,5-triazine: Relevance for photoacid generation. J. Am. Chem. Soc. 121, 6167-6175 (1999).

54. Kuang, X. et al. Grayscale digital light processing 3D printing for highly functionally graded materials. Sci. Adv. 5, eaav5790 (2019).

55. Zheng, X. et al. Design and optimization of a light-emitting diode projection micro-stereolithography three-dimensional manufacturing system. Rev. Sci. Instrum. 83, 125001 (2012).

56. Zheng, X. et al. Ultralight, ultrastiff mechanical metamaterials. Science 344, 1373-1377 (2014).

57. Han, D. et al. 4D printing of a bioinspired microneedle array with backwardfacing barbs for enhanced tissue adhesion. Adv. Funct. Mater. 30, 1909197 (2020).
58. Gong, H., Bickham, B. P., Woolley, A. T. \& Nordin, G. P. Custom 3D printer and resin for $18 \mu \mathrm{m} \times 20 \mu \mathrm{m}$ microfluidic flow channels. Lab Chip 17, 2899-2909 (2017)

59. Tang, T.-M., Pei, Y., Li, Z.-Y., Ni, H.-J. \& Wang, X.-X. Rapid prototyping manufacturing of trefoil knot based on Pro/E. Manuf. Autom. 37, 57-59 (2015).

60. Toba, Y., Usui, Y., Alam, M. M. \& Ito, O. Onium butyltriphenylborates as donor-acceptor initiators for sensitized photopolymerizations of vinyl monomer. Macromolecules 31, 6022-6029 (1998).

61. Stoll, S. \& Schweiger, A. Easyspin, a comprehensive software package for spectral simulation and analysis in EPR. J. Magn. Reson. 178, 42-55 (2006).

62. Peng, H. Y. et al. High performance graded rainbow holograms via two-stage sequential orthogonal thiol-click chemistry. Macromolecules 47, 2306-2315 (2014).

63. Han, D., Yang, C., Fang, N. X. \& Lee, H. Rapid multi-material 3D printing with projection micro-stereolithography using dynamic fluidic control. Addit. Manuf. 27, 606-615 (2019).

\section{Acknowledgements}

We thank the financial support from the NSFC (51773073 and 52073108), the HUST peak boarding program, and the Fundamental Research Funds for the Central Universities (2019kfyRCPY089). We also thank the technical assistance from the HUST Analytical \& Testing Center and Miss Min Lei at the Core Facilities of Life \& Sciences (HUST).

\section{Author contributions}

X.Z. conducted the experiments and density functional theory calculations, analyzed the results, and drafted the paper. Y.Z. helped to analyze the results. M.-D.L. performed the transient absorption experiments. Z.A.L. and T.X. participated in revising the paper. H.P. and X.X. supervised the project and edited the paper.

\section{Competing interests}

The authors declare no competing interests.

\section{Additional information}

Supplementary information The online version contains supplementary material available at https://doi.org/10.1038/s41467-021-23170-4.

Correspondence and requests for materials should be addressed to H.P. or X.X.

Peer review information Nature Communications thanks Ren Liu and the other, anonymous, reviewer(s) for their contribution to the peer review of this work. Peer reviewer reports are available.

Reprints and permission information is available at http://www.nature.com/reprints

Publisher's note Springer Nature remains neutral with regard to jurisdictional claims in published maps and institutional affiliations.

Open Access This article is licensed under a Creative Commons Attribution 4.0 International License, which permits use, sharing, adaptation, distribution and reproduction in any medium or format, as long as you give appropriate credit to the original author(s) and the source, provide a link to the Creative Commons license, and indicate if changes were made. The images or other third party material in this article are included in the article's Creative Commons license, unless indicated otherwise in a credit line to the material. If material is not included in the article's Creative Commons license and your intended use is not permitted by statutory regulation or exceeds the permitted use, you will need to obtain permission directly from the copyright holder. To view a copy of this license, visit http://creativecommons.org/ licenses/by/4.0/.

(C) The Author(s) 2021 